\section{A STUDY OF LATE POSTPARTUM ECLAMPSIA - 178 CASES}

Shahin Akhter Jahan Habib ${ }^{1}$, Sayeeda Sultana ${ }^{2}$

\begin{abstract}
:
Objective: The purpose of this study was to determine whether there is a shift in the timing of eclampsia in relation to delivery and whether traditional symptoms precede impending postpartum eclampsia.
\end{abstract}

Study Design: A cross - sectional hospital - based study conducted at Gynae Unit, Dhaka Medical College Hospital from January 2006 - December 2007.

Data was collected regarding the relationship of the patient's first seizure to delivery prodromal symptoms, neuroimaging studies, use of magnesium sulfate, antihypertensive therapy and follow up medical care. The analysis focused on women who had late postpartum eclampsia.

Result: During the study period 178 patients were diagnosed with eclampsia. Fifty eight women (33\%) had postpartum eclampsia, of which 46 women (79\%) had late onset (> 48 hrs.). Interestingly only 10 of these 46 women (22\%) had been previously diagnosed with preeclampsia .Forty two pt.(91\%) with late postpartum eclampsia had at least 1 prodromal symptom, and 24 pt. (52\%) had> 1 symptom that heralded the seizure : 40 women (87\%) had headache, 20 women (44\%) had visual changes , 10 (22\%) had nausea or vomiting and 4 (9\%) experienced epigastric pain. Only 14 of this 42 women (33\%) sought care for their symptoms, of whom 12 women (86\%)had clinical evidence of preeclampsia that was not considered by the treating physician. Among all patients with eclampsia there were 14 cases of aspiration pneumonia, 6 cases of pulmonary edema, 6 cases of pleural effusion 4 cases of DIC and no cases of maternal death.

Conclusion: Current obstetric treatment has a resulted in a shift of eclampsia toward the postpartum period with most cases being seen as late postpartum .To reduce the rate of the postpartum eclampsia, efforts should be directed to the education of the health care providers and patients regarding the importance of prompt reporting and evaluation of symptoms of preeclampsia during the postpartum period.

\section{Introduction:}

Eclampsia is serious obstetric complication1 that accounts for $15 \%$ maternal deaths in USA 2 . In certain populations, it is a major cause of maternal and perinatal morbidity and death.3-4 The onset of eclampsia can be antepartum, intrapartum, or

1. Assist Professor, Dept. of Obst \& Gyn, Dhaka Medical College, Dhaka

2. Assist Professor, Dept. of Obst. \& Gyn, BSMMU, Dhaka postpartum 4-5. In the postpartum period, the onset of convulsions has been seen within the first 48 hrs. and beyond $48 \mathrm{hrs}$. (Late postpartum) 6. In the western world, the rate of eclampsia has been declined as a result of universal institution of prenatal care, early detection of preeclampsia, timely delivary of women with severe preeclampsia, and the use of magnesium sulfate intrapartum and immediately after delivery 1,7-9. The objective of our report was to review 2 years of experience with eclampsia to determine whether current treatment protocols have resulted in any changes in this serious obstetric complication and to determine whether traditional symptoms can be used to predict the occurrence of eclampsia in the postpartum period

\section{Material and methods}

This was a 2 yrs. analysis of all pts. who were delivered from January 2006- December 2007, and who had eclamptic).6, convulsions and were cared for at the DMCH. Patients were identified through a perinatal database and through discharge diagnosis International Classification of Diseases, 9 th revision codes. Records were reviewed for pt. demographic information, the timing of first seizure in relation to delivery, associated prodromal symptoms, and lab. finding radiologic imaging, use of magnesium sulfate, antihypertensive therapy follow-up medical care, and associated morbidities .

Eclampsia was defined as the onset of convulsions in women with clinical signs and symptoms that were consistent with preeclampsia. Late postpartum eclampsia was defined as the onset of convulsions $>48 \mathrm{hrs}$. after delivery .10 Patients with late postpartum eclampsia were considered to have been previously diagnosed with preeclampsia in the index pg. if they had required treatment with magnesium sulfate for seizure prophylaxis . In the case of pts. Who were not delivered in our hospital, we routinely obtained details delivery course and documentation and reviewed.

In Patients with late postpartum eclampsia, neurodiagnostic imaging was preformed to rule out the presence of precipitating central nervous system pathologic conditions (such as infection, tumors, central nervous system hemorrhage, and thrombosis).6 Eclampsia was deemed to be preventable if the patient sought medical care for sings or symptoms that were typical of preeclampsia but did not receive timely appropriate evaluation or treatment for preeclampsia. In addition, eclampsia was considered possibly preventable if the patient had persistent headaches, blurred vision, epigastric pain, nausea, or vomiting that were not reported to health care providers because the patient did not consider these symptoms to be serious. Our analysis will focus on the group of patients who had postpartum eclampsia. 
Statistical analysis. Comparison of frequencies was performed with x2 test, where appropriate; odds ratios (oRs) and 95\% CIs were calculated with the Mantel- Haenszel method. All statistical analyses were performed with the SAS statistical software package.

\section{Results:}

During the study period, 98 patients with eclampsia were cared for at our institutions. Most of the patients were delivered at our centers, although 10 of the 23 patients (43.5\%) with late onset postpartum eclampsia were delivered elsewhere. Table I describes the demographic characteristics of the study patients. The mean maternal age was 21.2 years (range, $13-40$ years).

Table-I: Patient Demographic Information

\begin{tabular}{lcc}
\hline Demography & No & $\%$ \\
\hline Nulliparous & 120 & 67.4 \\
PreviousPreeclampsia & 14 & 7.9 \\
Chronic hypertension & 14 & 7.9 \\
Diabetes Mellitus & 6 & 3.4 \\
\hline
\end{tabular}

Table II describes the time of onset of convulsions in relation to delivery. Among the 6 women who had early postpartum eclampsia, 4 women (66.7\%) were diagnosed with preeclampsia before delivery. One patient had not received magnesium sulfate at the time of onset of convulsions, whereas 1 patient had convulsions while being treated with magnesium; the other 2 women had completed 24 hours of magnesium therapy. The remaining two women had postpartum eclampsia despite the absence of any clinical evidence of antepartum of intrapartum pre eclampsia. In contrast, only 5 of the women (21.07\%) with late postpartum eclampsia had been previously diagnosed with preclampsia (OR 7.2, 95\% CI 1.0-51.4).

Table-II: Timing of onset of convulsions relative to delivery

\begin{tabular}{lcc}
\hline Timing of eclamosia & No & $\%$ \\
\hline Befor delivery & $120 / 178$ & 67.4 \\
Post partum & $58 / 178$ & 32.6 \\
$<48$ hours & $12 / 58$ & 20.7 \\
$>48$ hours* & $46 / 178$ & 79.3 \\
\hline
\end{tabular}

Table III compares the frequency of maternal symptoms before the development of convulsion between those women who had eclampsia before delivery or immediately after delivery and those women who had late-onset postpartum eclampsia. Women with late postpartum eclampsia were more likely to have headaches (OR, 4.1), visual symptoms (OR, 2.6), and at least 1 symptom of preeclampsia (OR, 4.6), compared with the other women with eclampsia. Two or more symptom were present in 12 of 23 women (52.2\%) with late onset eclampsia, which was not different from the rate (27 of 66 women [40.9\%]) in patient with earlier onset convulsions $(p=.348)$.

Table-III: Prodromal symptoms in patients with late postpartum eclampsia

\begin{tabular}{lccc}
\hline $\begin{array}{l}\text { Timing of } \\
\text { eclampsia }\end{array}$ & $\begin{array}{c}\text { Late } \\
\text { postpartum } \\
\text { eclampsia } \\
(\mathrm{No})\end{array}$ & $\begin{array}{c}\text { Before } \\
\text { delivery or early } \\
\text { postpartum } \\
(\mathrm{No}) *\end{array}$ & \\
\hline Headache & $40(87.0 \%)$ & $82(62.1 \%)$ & $4.1(1.1,15.1)$ \\
Visual symptoms & $20(43.5 \%)$ & $30(22.7 \%)$ & $2.6(1.0,7.2)$ \\
Nausea or vomiting & $10(21.7 \%)$ & $34(25.8 \%)$ & $0.8(0.3,2.5)$ \\
Epigastric pain & $4(8.7 \%)$ & $18(13.6 \%)$ & $0.6(0.1,3.0)$ \\
At least 1 & $42(91.3 \%)$ & $92(69.7 \%)$ & $4.6(1.0,21.4)$ \\
\hline
\end{tabular}

Only 7 of the 21 women (33.3\%) who had symptoms of preeclampsia sought medical care for their complaints. All these women received evaluation in the emergency department. Indeed 2 women had gone to the emergency department on 3 separate occasions with server headache before having convulsions. Sis of the 7 patent were judged to have had preventable seizures on the basis of failure to consider or suspect a diagnosis of preeclampsia in the presence of the hypertension ( $\mathrm{n}=6$ women) and symptom consistent with preeclampsia. In addition, laboratory testing was not obtained in most of these patients. The seventh patient was admitted to the obstetric service with isolated nausea and vomiting. Because she remained normotensive and nonproteinuric and had unremarkable laboratory evaluation, her convulsions were not considered to have been preventable. The remaining 14 women did not consider their symptoms serious enough to warrant medical evaluation.

All women with late-onset postpartum eclampsia received neurodiagnostic imaging. Only one of the 23 women had significant findings: a transverse sinus thrombosis identified on magnetic resonance venography. We would also consider her convulsions nonpreventable.

Table IV describes the maternal complications among all eclampsia women. The most common occurrence was aspiration pneumonia. Five patients required blood transfusion. There were no maternal deaths. The average length of stay was 6.8 days (range, 2-30 days). 
Table-IV: Maternal complications among all patients with eclampsia

\begin{tabular}{lcc}
\hline Complication & No. $(\mathrm{n}=178)$ & $\%$ \\
\hline Aspiration pneumonia & 14 & 7.9 \\
Pulmonary edema & 6 & 3.4 \\
Pleural effusion & 6 & 3.4 \\
Disseminated intravascular coagulation & 4 & 2.2 \\
Retinal detachment & 2 & 1.1 \\
\hline
\end{tabular}

\section{Comment:}

During the past decade, several studies have described the clinical signs and symptoms in women before the development of eclampsia $1,5,7,11$ in these studies; attempts were made to identify risk factors that could be helpful in the prevention of some of these eclampsia cases. In general, all he aforementioned studies have noted a changing pattern of eclampsia during the fist decades. Latcheta $\mathrm{al}^{7}$ evaluated changes in the incidence and pattern of eclampsia within he same geographic area over a 60-year period.

The current study was designed to determine the pattern and morbidity of eclampsia areas in the United States. The findings of this study revealed that one third of the cases of eclampsia developed after delivery, with most of the postpartum cases (79\%). Overall, 26\% of all cases of eclampsia in this study developed beyond 48 hours after delivery. This rate is higher than those reported by Douglas and Redman (5.2\%) ${ }^{1}$ and Kats et al (5.6\%). ${ }^{11}$

The marked reduction in he incidence of eclampsia over the years can be attributed to prenatal care, early detection of signs and symptoms of preeclampsia, and prophylactic use of magnesium sulfate during labor and after delivery in women with preeclampsia. 1,5,7,8 These changes were brought about through continuous education of both patients and health care providers regarding the prompt reporting and response to serious symptoms such as severe headaches, blurred vision, mental status changes, and epigastic pain with nausea and vomiting. Unfortunately, such efforts at the education of our patients have not been continued into the postpartum period, yet these women remain at risk. It is indeed troubling to note that $91 \%$ of women had at least I symptom suggestive of preeclampsia before the onset of convulsions, yet only 33\% of women reported these symptoms to a health care provider. This suggests that there is an urgent need to focus our efforts at patient education regarding what symptoms to report after delivery. At the time of discharge from the hospital, women must be instructed that, although they have delivered, they may still be at risk for preeclampsia and that they should remain aware of symptoms of the disease, which should include verbal instruction, and possibly a printed instruction sheet such as that suggested by Barton et al, 12 indeed, these education efforts must be directed to all patients, regardless of risk factors. Katz et al ${ }^{11}$ reported that, among 53 cases of eclampsia, only 7 women (13\%) were diagnosed with server preeclampsia before convulsions. Our experience in the late postpartum period was similar because only $22 \%$ of these women had a history of preeclampsia during the index pregnancy, and most of our patients with late convulsions did not have exclusive risk factors for preeclampsia.

In general, women in whom symptoms develop beyond 48 hours after delivery usually are seen in the emergency department. Because our colleagues in the emergency department will be the fast providers to evaluate such women, it is vital that we educate them that a high index of suspicion remains important after delivery, as dose a low threshold for early involvement of the obstetric staff. If we fail to educate physicians regarding the importance disturbing to not that 6 cases of eclampsia might have been prevented if preeclampsia had been considered during the evaluation of these women.

Among the 14 women who had postpartum symptoms that were not reported to a health care provider, it is difficult to know that proportion of these cases could have been prevented had these women sought medical attention. Further studies are needed to address this question.

The limitation of this study lies in its retrospective nature and inclusion of patients on the basis of discharge diagnosis coding. If the diagnoses were not coded correctly, patients may have been unintentionally excluded. In addition, although we believe that our findings represent a decrease in intrapartum and early postpartum eclampsia with a resultant increase in the proportion of eclampsia in the late period, they may simply reflect improved identification of late onset postpartum eclampsia , which this study was not designed to detect.

Finally, if symptoms have not been reliably recorded in the patient record, our results may be affected. Indeed, this may be reflected in a lower occurrence of headache, visual symptoms, and epigastric pain among patients with eclampsia before delivery than were previously reported by Sibai et al. ${ }^{13}$ We do not believe, however, that this possibility affects our conclusion that prodromal symptoms will help us to prevent late onset postpartum eclampsia, because the occurrence of symptoms was higher in this group of patients. With current obstetric treatment protocol, we have documented an increasing rate of eclampsia in the late postpartum period. By directing efforts at educating patients and health care providers in the continued monitoring, reporting, and prompt evaluation of symptoms in the postpartum period, we believe that, in some patients, late postpartum eclampsia can prevented. 


\section{References}

1. Douglas KA, Redman CW. Eclampsia in the United Kingdom. BMJ 1994; 309:1395-400.

2. American College of Obstetricians and Gynecologists. Hypertension in pregnancy. Washington (DC): The College, 1996. Technical Bulletion No.: 219.

3. The Clampsia Trial Collaborative Group. Which anticonvulsant for women with eclampsia? Evidence from the Collaborative Eclampisa Trial [published erratum appears in Lancet 1995;946;258]. Lancet 1995;945:145563.

4. Lopez-Llera MM. Main clinical types and subtypes of eclampsia. Am J Obstet Gynecol 1992;166:4-9.

5. Mattar F, Sibai BM. Eclapsia, VIII. Risk factors for maternal morbibity. Am J Obstet Gynecol 2000;182:307-12.

6. Lubarsky SL, Barton JR, Friedman SA, Nasreddine S, Ramddan MK, sibai BM . Late postpartum eclampsia revisited. Obstet Gynecol 1994;83:502-5.
7. Leitch CR, Cameron AD, Walker JJ. The changing pattern of eclampsia over a 60 - year period Br J Obstet Gynaecol 1997;104:917-22

8. moller B, Lindmark G.Eclampsia in Sweden, 1976-80. Acat Obstet Gynecol Scand 1986;65:307-14.

9. Saftlas AF, Olson DR, Franks AC, Atrash HK, Polaras R. Epidemiology of preeclampsia and eclampsia in the Inited States, 1979-1986. Am J Obstet Gynecol 1990; 163:460-5.

10. Sibia BM, Schneider JM, Morrison eclampsia controversy Obstet Gynecol 1980; 55:74-8.

11. Katz VL, Farmer R, Kuller J. preeclampsia into eclampsia: toward a new paradigm. Am J Obstet Gynecol 2000; 182:1389-96

12. Barton JR, Witlin AG,Sibai BM.Management of mild preeclampsia. Clin Obstet Gynecol 1999; 42:455-69.

13. Sibai BM, McCubbin JH, Anderson GD, Lipshitz J,Dilts PV Jr.Eclampsia. I.Observations from 67 recent cases. Obstet Gynecol 1981; 58:609-13. 